\title{
Computing Stochastical Bounds for the Tail Distribution of an M/GI/1 Queue
}

\author{
Pierre L. Douillet ${ }^{1}$, André-Luc Beylot ${ }^{2}$, and Monique Becker ${ }^{3}$ \\ 1 Mathématiques Supérieures, CPGE Faidherbe, \\ 9, rue A. Carrel, 59000 Lille, France \\ douillet@cnam.fr \\ http://www-inf.int-evry.fr/ douillet/ \\ 2 Laboratoire PRiSM, Université de Versailles, \\ 78035 Versailles Cedex -France \\ beylot@prism.uvsq.fr \\ 3 Institut National des Télécommunications, \\ rue Charles Fourrier 91011 Evry Cedex, France \\ mbecker@etna.int-evry.fr
}

\begin{abstract}
Packet switching networks lead mostly to M/GI/1 queue models. In this paper, computing methods are designed in order to get quickly approximate values for response time of these networks. Laplace transform is a powerful tool to study such queuing systems. But inversion of the Laplace transform on the real line is well known to be an ill-conditioned problem and usual numerical methods of inversion fail to give accurate error bounds.

A new method to address this old problem is afforded by the recently developed formal computing tools: exact computations can be done during the first steps of calculation, while usual floating point computations remain confined to the last steps. Applying that method to an M/GI/1 queue, a formal approach is designed, leading to proven bounds, and several numerical improvements are proposed. Accurate bounds are obtained.
\end{abstract}

\section{Introduction}

The M/GI/1 queue is the most useful model for packet switching networks. It is often realistic to assume that packet arrival process is Poisson, but service time is not exponential at all. Services are independent when treatments depend upon the packet length, and when this length is constant, the adequate model is $\mathrm{M} / \mathrm{D} / 1$. Otherwise $\mathrm{M} / \mathrm{GI} / 1$ queues are used.

In this paper are considered known results [1 about M/GI/1 queue, but from a new point of view, allowed by an efficient use of formal computing. Efficient algorithms are designed in order to obtain bounds on performance criteria for such a queue. The tail of the response time distribution of such an M/GI/1 queue can be evaluated from Pollaczek-Khintchine formula, leading to deal with Laplace transforms. 
But when dimensioning systems, Laplace transforms have to be inverted, and the usual numerical methods give approximations for which accurate error bounds are not available. This is rather not a new subject [2] [3] [5], but a new method to attack this old problem can be obtained as follows.

We have written, in Maple code, a package of procedures that computes the formal convolution of two piecewise polynomial functions (i.e. splines). This is especially usefull when dealing with inverse Laplace transform, since such a computation is strongly ill-conditioned. A method using exact computations during the first steps of iterative algorithms is therefore significantly more accurate than any other method. From these formal computations, two kinds of results concerning the sojourn-time pdf can be obtained: exact bounds for the first part of the curve and an accurate approximation of the second part of the curve. Moreover, unlike other algorithms, the proposed algorithm leads to an error term that is not an oscillating function, and requires no "calibrations".

The present paper is organized as follows: assumptions and notations are presented in section 1. The inversion of the Pollaczek-Khintchine formula is reminded in section 2. Two formal approaches are designed in sections 3 and 4 . Numerical improvements are proposed in section [5] Conclusions are formulated in section [6] and the paper ends with some references.

\section{Assumptions and Notations}

Let us use the following notations. When $\mathbf{X}$ is some random variable, its pdf (probability density function) will be noted $x$, its cdf (cumulative distribution function) will be noted $X$ and its Laplace transform will be noted $\widehat{x}$. In other words $x(u) d u=\operatorname{Pr}\{\mathbf{X} \in[u, u+d u]\}, X(u)=\int_{0}^{u} x(t) d t$, and $\widehat{x}(z)=$ $\int_{0}^{u} x(t) \exp (-z t) d t$.

Let $\mathbf{A}$ be the random variable "inter-arrival duration" and $\lambda=1 / \mathbf{E}(\mathbf{A})$, so that: $a(t)=\lambda \exp (-\lambda t)$. Let $\mathbf{B}$ be the random variable "service duration" and $\mu=1 / \mathbf{E}(\mathbf{B})$. This last value is the average throughput during busy periods, and the quantity $\rho=\lambda / \mu$ is the load of the system. It is assumed that no infinite queue happens (therefore $\rho<1$ ).

The random variable $\mathbf{R}$ will denote the sojourn time of a customer in the system, $\mathbf{W}_{0}$ its (perhaps being null) waiting time, and $\mathbf{W}_{1}$ its conditional waiting time, i.e. the waiting time knowing that the customer will wait. All the preceding random variables, and especially the service duration $\mathbf{B}$, are "individual variables", i.e. can be perceived as the result of a process that picks a customer by uniform sorting over their order of arrival, and notes the value associated to this customer.

Another process of selection can be used, that picks a customer by uniform sorting over the instants of observation (that sorting being repeated until a client is eventually served), leading to "temporal" variables. Let $\beta$ be the pdf of $\widetilde{\mathbf{B}}$ (the temporal service duration). Relation $\beta(t)=\mu t b(t)$ is obvious and gives $\mathbf{E}(\widetilde{\mathbf{B}})=(1 / \mu)+\mu \operatorname{var}(\mathbf{B})$, while the variance of $\widetilde{\mathbf{B}}$ includes the third moment of $\mathbf{B}$. 
By definition, a customer arriving in a non-empty system finds another customer that is currently being served (and maybe other ones, being waiting). The duration that the incoming customer has to wait until the served customer leaves the system is called the "residual service time", and will be noted $\mathbf{C}$. The pdf of $\mathbf{C}$ is well-known to be $c(t) d t=\mu d t \int_{u=t}^{u=\infty} b(u) d u$, and we have $\mathbf{E}(\mathbf{C})=\frac{1}{2} \mathbf{E}(\widetilde{\mathbf{B}})$, while $\operatorname{var}(\mathbf{C})$ involves up to the third moment of $\mathbf{B}$. In what follows, the notations $\bar{x}$ and $\sigma^{2}$ will always refer to $\mathbf{E}(\mathbf{C})$ and $\operatorname{var}(\mathbf{C})$.

\section{Inversion of the Pollaczek-Khintchine Formula}

\section{$2.1 \quad$ Introduction}

It is well known that the Laplace transforms of the sojourn time $\mathbf{R}$ and the service duration $\mathbf{B}$ are related by the Pollaczek-Khintchine formula: $\widehat{r}(z)=$ $\frac{(1-\rho) z \widehat{b}(z)}{z-\rho \mu+\rho \mu \widehat{b}(z)}$. But the residual service time obeys to $\widehat{c}(z)=\mu \frac{1-\widehat{b}(z)}{z}$, and we have:

$$
\widehat{r}(z)=\widehat{b}(z) \frac{(1-\rho)}{1-\rho \widehat{c}(z)}
$$

Since the Laplace transform of the sum of two independent r.v.'s is the product of the Laplace transforms of these r.v.'s, (1) leads to $\widehat{w_{0}}(z)=\frac{1-\rho}{1-\rho \widehat{c}(z)}$, the sojourn time of a customer being the sum of his waiting time and his service duration.

The computation of $\widehat{w_{1}}$ may be derived when noticing that a conditional waiting time is the sum of a residual service duration and an ordinary waiting time, leading to $\widehat{w_{1}}=\widehat{c} \widehat{w_{0}}$. It may also be noticed that an ordinary waiting time is either null (with probability $1-\rho$ ) or is a conditional waiting time (with probability $\rho$ ), leading to $\widehat{w_{0}}=(1-\rho)+\rho \widehat{w_{1}}$, i.e. to the same result.

\subsection{An Inverse Transform Method}

The power-series expansion of (1) is: $\widehat{r}(z)=(1-\rho) \widehat{b}(z) \sum_{k>0}(\rho \widehat{c}(z))^{k}$ and these series converge (when $z$ is in the right-hand half-plane, i.e. $\Re(z) \geq 0$ ) since $c$ verifies: $|\widehat{c}(z)|=\left|\int_{0}^{\infty} c(t) \exp (-z t) d t\right| \leq\left|\int_{0}^{\infty} c(t) d t\right|=1$ and $\rho<1$. Therefore we have:

$$
r(t)=b(t) \otimes \sum_{k \geq 0}\left(\rho^{k} c[k](t)\right) / \sum_{k \geq 0} \rho^{k}
$$

where $\otimes$ is the convolution operator, $c[k](t)$ is the convolution of $k$ functions equal to $c(t)$ and therefore $c[0](t)=\operatorname{Dirac}(t)$. That formula can be rewritten as:

$$
\begin{aligned}
r(t) & =(1-\rho) b(t)+\rho b(t) \otimes w_{1}(t) \\
w_{1}(t) & =\sum_{k \geq 1}\left(\rho^{k} c[k](t)\right) / \sum_{k \geq 1} \rho^{k}
\end{aligned}
$$


Equation (3) says again that the sojourn time is equal to the service time when a customer arrives in an empty queue (which happens with probability $1-\rho$ ) and otherwise is increased by the conditional waiting time. Equation (4) says that the pdf of the conditional waiting time may be obtained by a disjunction into an infinite number of cases, each one occurring with probability $(1-\rho) \rho^{k-1}$ and corresponding to the sum of $k$ independent residual service times [7]. Let us note $r[n]$ and $w_{1}[n]$ the functions obtained by truncating the summations in both numerator and denominator of (2) and (4). These functions have again a unit mass, i.e. are again the pdf of some random variables.

\subsection{Example of Deterministic Services}

Let us consider a deterministic server with $\rho=0.75, \mu=0.1$. In other words, $c(t)=\mu=0.1$ when $t<10$ and $c(t)=0$ otherwise. After some computations [8], the convolutions $c[2] \ldots c[16]$ of the residual service time pdf can be obtained, leading to Fig. 1

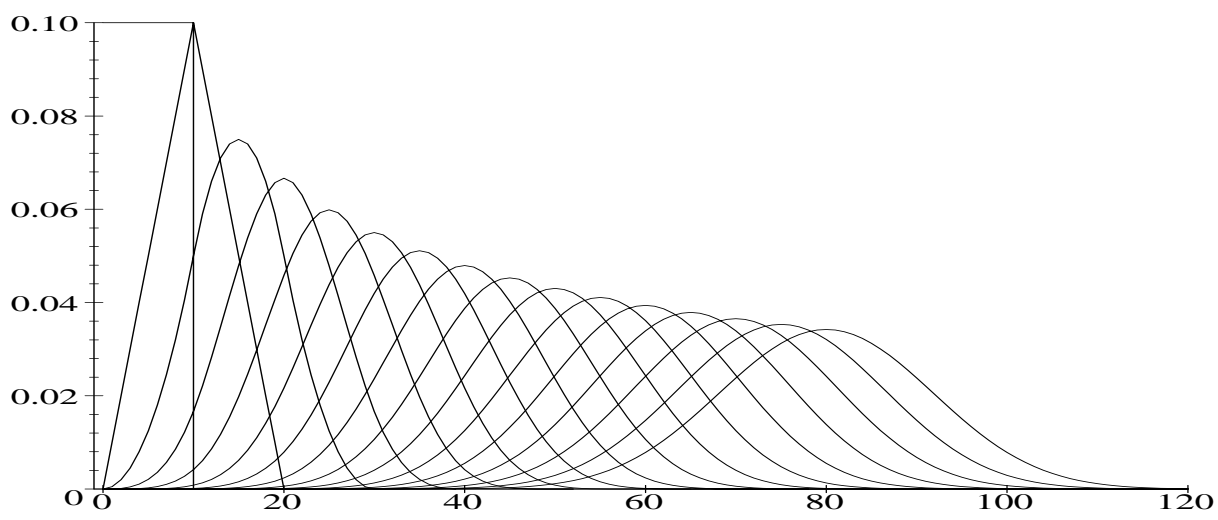

Fig. 1. M/D/1 file: from $c[2]$ to $c[16]$.

These functions become more and more regular, while flattening on the horizontal axis and sliding towards right. This sliding is due to $\mathbf{E}(c[n])=n \mathbf{E}(\mathbf{C})=$ $n \bar{x}$, while flattening is due to $\operatorname{var}(c[n])=n \operatorname{var}(\mathbf{C})=n \sigma^{2}$ : when the curve gets wider, its height has to decrease, since the area underneath the curve is constant. Getting regular is nothing but the central limit theorem, i.e. the convergence of the reduced pdf of a sum of i.i.d. variables towards the normal law.

Figure 2 shows the approximations $w_{1}[3] \ldots w_{1}[16]$ of the conditional waiting time distribution. It appears that those functions converge to a limit function and that convergence is faster for small values of $t$. More precise statements about this convergence will be given in next section. 


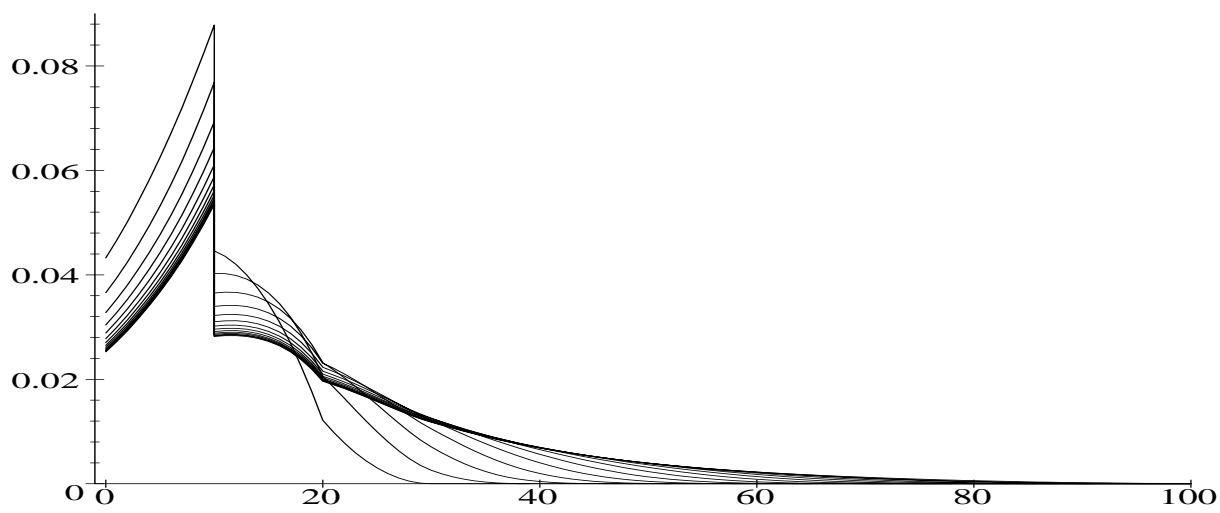

Fig. 2. M/D/1 file: from $w_{1}[3]$ to $w_{1}[13]$.

\section{First Bounds}

\subsection{Statement and Illustration}

According to our general notations, $R[n](t)$ will denote $\int_{0}^{t} r[n](u) d u$. A first claim is:

$$
\forall n:\left(1-\rho^{n+1}\right) R[n] \leq\left(1-\rho^{n+2}\right) R[n+1] \leq R \leq R[n+1] \leq R[n]
$$

As an illustration of this theorem, the left part of Fig. 3 shows the sequence of the lower bounds relative to and for an $\mathrm{M} / \mathrm{D} / 1$ queue where the right part shows the related upper bounds.
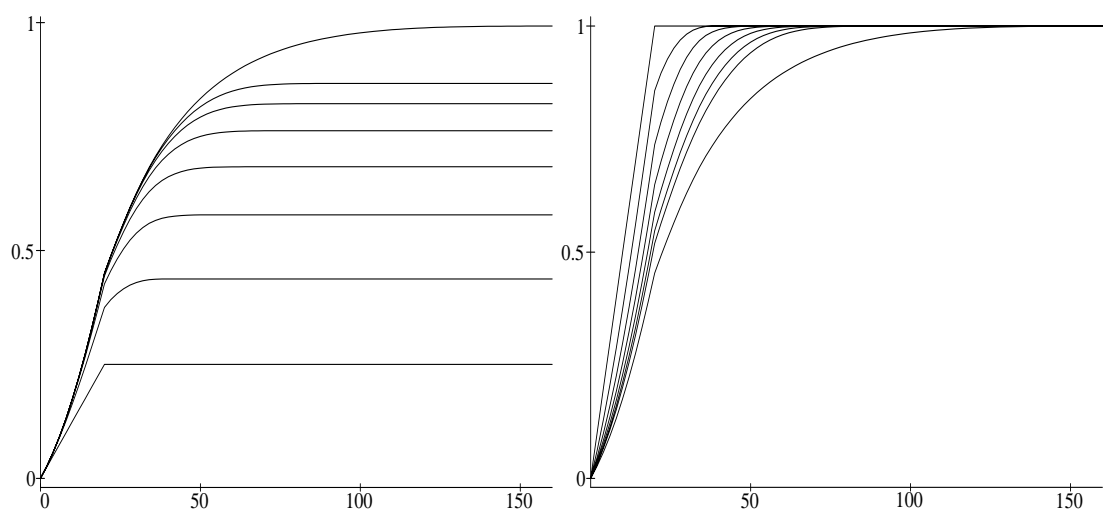

Fig. 3. M/D/1 file: cdf lower (left) and upper (right) bounds 


\subsection{Proof}

For the left part: from the very definition of $r[n],\left(1-\rho^{n+1}\right) r[n](t)$ equals $\sum_{n \geq k} \rho^{k} b(t) \otimes c[k](t)$. Convolution products of $c$ and $b$ are pdf's, so every term in this sum is positive and the sequence of functions $\left(1-\rho^{n+1}\right) r[n](t)$ is increasing. This remains true when integrating, so the sequence of functions $\left(1-\rho^{n+1}\right) R[n](t)$ is also increasing .

For the right part: each $c[k]$ is the pdf of a r.v. $\mathbf{X}_{k}$, equal to the sum of $k$ i.i.d. variables $\mathbf{X}_{1, k} \ldots \mathbf{X}_{k, k}$, each one being distributed according to $c$. The function $w_{0}[n]$ can be viewed as the pdf of the r.v. $\mathbf{Y}_{n}$ defined by: pick a $k \in[0, n]$ according to weight $\rho^{k}$ (i.e. with a probability proportional to $\rho^{k}$ ) and then pick a $\mathbf{X}_{k}$. Therefore, $1-R[n](t)$ is the probability that $\mathbf{B}+\mathbf{Y}_{n} \geq t$ while $1-R[n+1](t)$ is the probability that $\mathbf{B}+\mathbf{Y}_{n+1} \geq t$. Since the odds of the former are obviously lower than those of the latter, the sequence $R[n]$ is decreasing.

\subsection{Limitations of This Result}

The bounds given in (5) are simple, and apply to the whole scale of response times. But their efficiency has to face two kinds of remarks. Firstly, and it can be easily seen on Fig. 3 the exact curve is not at the same distance from the two bounds, starting very near to the lower bound and ending very near to the upper bound, giving the limit value $R(\infty)=1$.

Secondly, these bounds provide nearly no information about the tail of the distribution. Let us for example consider a deterministic law. It may easily be seen that residual service duration pdf is: $c(t)=\mu \chi_{[0 ; 1 / \mu]}$ where $\chi$ is the usual indicator function ( 1 inside, 0 outside). Therefore $c[k]$ is zero outside $[0 ; k / \mu]$ and $R[k](t)=1$ when $t \geq k / \mu$. So let us look for more efficient bounds.

\section{Better Bounds}

\subsection{Statement and Illustration}

Let us now prove:

$$
\begin{aligned}
& \forall n: \quad\left(1-\rho^{n+1}\right) w_{1}[n] \leq\left(1-\rho^{n+2}\right) w_{1}[n+1] \leq w_{1} \\
& \forall n: \exists T_{n}: \forall t \leq T_{n}: w_{1}(t) \leq w_{1}[n+1](t) \leq w_{1}[n](t)
\end{aligned}
$$

As an illustration of this theorem, Fig. 4 (left) shows the sequence of the lower bounds relative to $n=1 . .8$ and $n=16$ for an M/D/1 queue (i.e. for a deterministic service distribution) where $\rho=0.75 ; \mu=0.1$, while Fig. 4 (right) shows some $T_{n}$ values.

Inequality (6) may be proved in the same way as the left hand inequality in (5). It leads to a global lower bound of the conditional waiting time pdf. The only existence of a $T_{n}$ satisfying inequality (17) is trivial $\left(T_{n}=0\right.$ works !): what is to be proved is that an optimal value can be derived and that the corresponding $T_{n}$ is meaningful. 

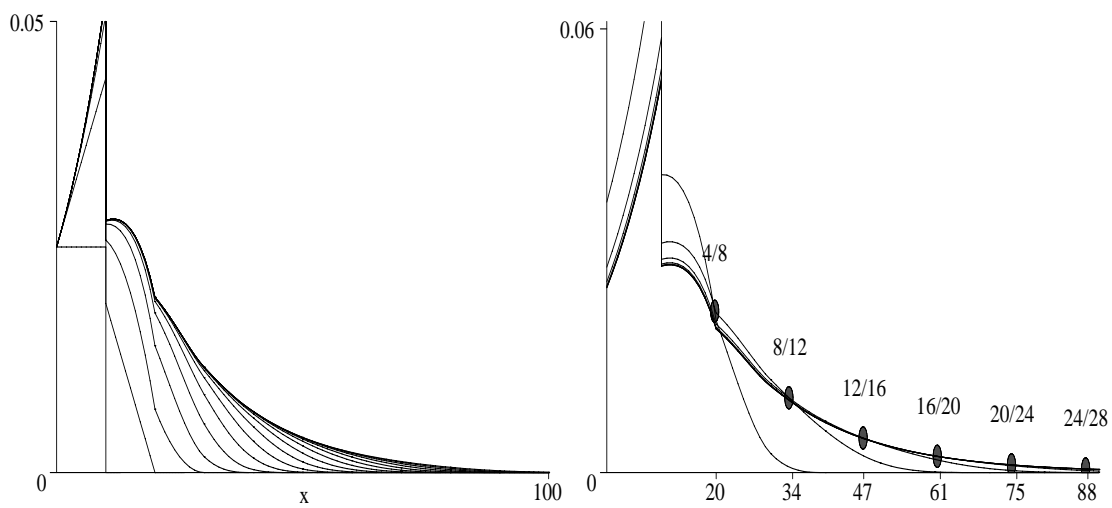

Fig. 4. Left: lower bounds. Right : upper bounds.

\subsection{Asymptotic Approximation}

Let us begin by computing the coordinate $T_{n, n+j}$ where the two curves $w_{1}[n]$ and $w_{1}[n+j]$ intersect. Figure 5 plots $T_{n, n+1}$ and $T_{n, n+4}$ against $n$ : the resulting graph appears as almost rectilinear. Computing the regression line (M/GI/1 file, with the given values of parameters) leads to $T_{n, n+1} \approx 3.468+3.401 n$ and $T_{n, n+4} \approx 6.032+3.406 n$. Each of these regression lines is "strongly explicative", since it reduces the standard deviation of the $T_{n}$ 's by a factor 300 .

Observing that $x \geq T_{n, n+j}$ induces $w_{1}[n](x) \geq w_{1}[n+j](x) \geq w_{1}(x)$, these regression lines can be used to choose the number of requested iterations to obtain reliable bounds on a given initial interval. With the parameters' values that were chosen in the example, it appears that exact bounds on the $[0,200]$ interval are obtained when $n=57$. Using this value leads to a relative error less than $\rho^{57} \approx 10^{-6}$. Therefore, events whose probability is more than $10^{-5}$ are almost exactly described (this probability corresponds to $x=200$ ).

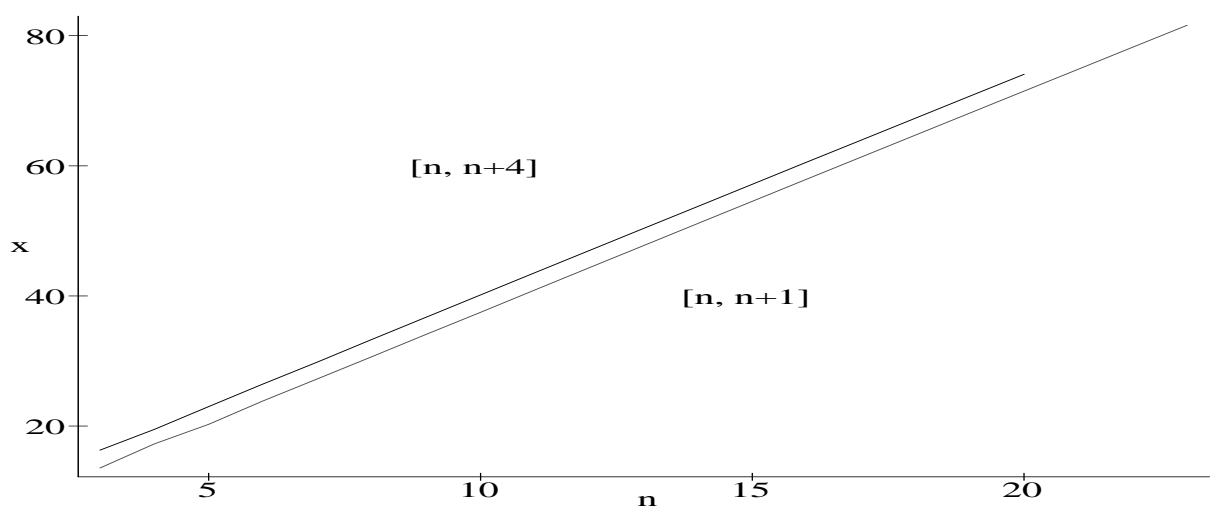

Fig. 5. Plotting $T_{n, n+1}$ and $T_{n, n+4}$ against $n$. 

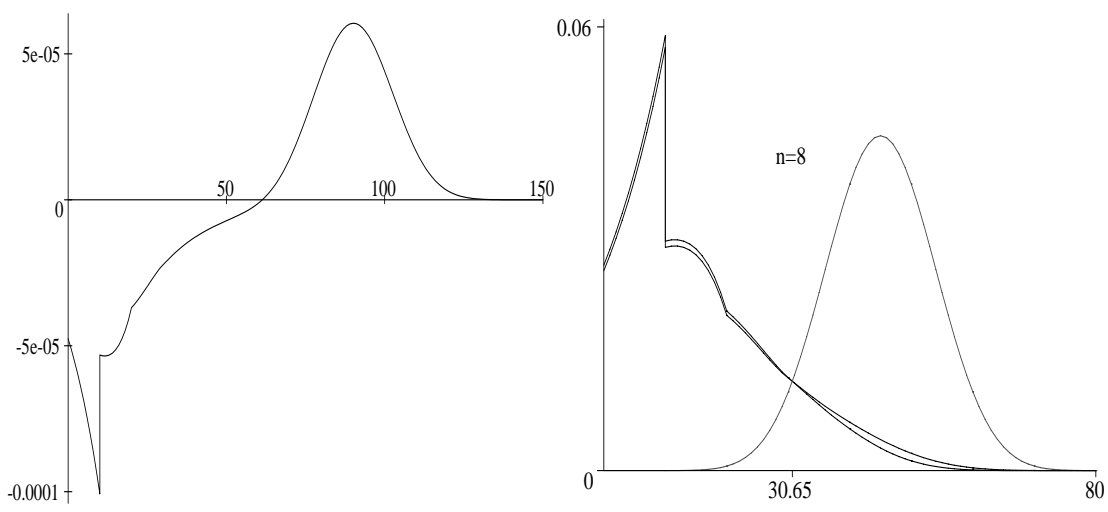

Fig. 6. Left: $w_{1}[18]-w_{1}[17]$. Right $: w_{1}[8], w_{1}[9]$ and $c[9]$.

\subsection{Proof}

To prove the existence and unicity of an abscissa $T_{n, n+1}$ such that $w_{1}[n](x)=$ $w_{1}[n+j](x)$, the main point is to observe that the difference between two successive $w_{1}[n]$ functions goes from negative values to positive values only once (Fig. 6, left).

This situation can be derived from the fact that $w_{1}[n+1]-w_{1}[n]$ is proportional to $c[n+1]-w_{1}[n]$, and therefore we are intersecting $w_{1}[n]$ and $c[n+1]$. By the way (Fig. 6, right), we obtain a better conditionned numerical equation. More precisely,

$$
w_{1}[n+1](t)-w_{1}[n](t)=\frac{(1-\rho) \rho^{n}}{1-\rho^{(n+1)}}\left(c[n+1](t)-w_{1}[n](t)\right)
$$

For increasing values of $n, c[n]$ becomes indistinguishable from nlaw $(\bar{x} n, \sigma \sqrt{n})$, the normal law with mean $n \mathbf{E}(\mathbf{C})=n \bar{x}$ and variance $n \operatorname{var}(\mathbf{C})=n \sigma^{2}$, while $w_{1}[n]$ converges towards $w_{1}$. But, for sufficiently large $t, w_{1}$ is indistinguishable from an exponential law (large deviation theorem). When $c$ is zero outside some finite domain, we have the more precise result $: w_{1}(t) \approx w_{\text {large dev }}(t)$ when $t \rightarrow \infty$ where $w_{\text {large dev }}(t) \doteq \sum_{k \geq 1}\left(\rho^{k}\right.$ nlaw $\left.(\bar{x} n, \sigma \sqrt{n})(t)\right) / \sum_{k \geq 1} \rho^{k}$. One obtains:

$$
w_{\text {large dev }}(t) \approx \frac{1-\rho}{\rho} \frac{1}{\eta} \exp \left(-\frac{\eta-\bar{x}}{\sigma^{2}} t\right) \quad \text { where } \quad \eta=\sqrt{\bar{x}^{2}-2 \ln (\rho) \sigma^{2}}
$$

In our example, numerical tests show that (8) is efficient as soon as $t \geq 10 \bar{x}$.

Therefore, intersecting $w_{1}[n]$ and $w_{1}[n+1]$ is equivalent to intersect a fixed (exponential) curve decreasing to 0 and a moving to right (bell) curve increasing from 0 , proving the existence and unicity of $T_{n}, n+1$ and the relation $T_{n+1, n+2}>$ $T_{n, n+1}$. Combining these relations, we obtain that, for a fixed $n$, the sequence $T_{n, n+j}$ is increasing. Its limit is the requested $T_{n}$. 
The core of the preceding proof being decreasing versus increasing (and not exponential versus normal), the result still holds for small values of $n$ (without requiring a formal proof, since a simple examination of the curves is sufficient).

\subsection{About Complexity}

The computation from convolution of functions $r[n], R[n], c[n]$ has a cost, that needs to be evaluated carefully. For M/D/1 queues, the $w_{1}[n]$ are piecewise polynomial functions $(n+1$ parts of degree $n-1)$. For the first iterations, it is efficient to use fractional numbers, while for the next ones, it is necessary to use floating point numbers. Then it is necessary to use a sufficient precision in order to avoid instability problems due to inversion of Laplace transform [6].

We used up to 100 decimal digits (which let us get an accuracy of only 5 useful digits for the final result). Programming carefully and using recent computers allow to compute convolutions of piecewise functions up to 100 pieces, each of degree 100, but not more.

\section{Two Improvements}

\subsection{Smoothing and Fast Convolution}

Let us see now two techniques giving a better approximation of $w_{1}$ beyond $T_{n}$. First an approximate value of $w_{1}[2 n]$ may be obtained from $w_{1}[n]$ by a fast exponential technique based on the following formula:

$$
\sum_{k=1}^{k=2 n} \rho^{k-1} c[k]=\sum_{k=1}^{k=n} \rho^{k-1} c[k]+\rho^{n} c[n] \otimes \sum_{k=1}^{k=n} \rho^{k-1} c[k]
$$

Let us note that a direct application of this formula does not improve the computation complexity. On the contrary it increases it. Of course, there are fewer convolutions to compute, but each of them takes into account functions which have a larger number of pieces, made of polynomials with higher degree: the number of integrations is higher and so is computing time. For a computation based on formula (9) to be possible, smoothing of $c[n]$ and $w_{1}[n]$ functions is necessary so as to decrease both the degree and the number of pieces.

An experimental study shows that applying (9) to the functions obtained by smoothing an exactly known $w_{1}[n]$ yields an important gain. But this method cannot be applied again, since the second smoothing, again needed to lower both degree and number of pieces, ruins the quality of approximation.

\subsection{Again, the Central Limit Theorem}

Another technique for approximating $w_{1}[2 n]$ may be obtained as a consequence of central limit theorem: $c[k]$ functions, when centered and reduced to a unit variance converge to a normal distribution, since a convolution of pdf's leads to 
the pdf of the sum of r.v.'s. Therefore, an approximation of even indexed $w_{1}$ 's is obtained by writing that $w_{1}[n](n \bar{x}+\tau \sqrt{n} \sigma) \approx \sqrt{2} w_{1}[2 n](2 n \bar{x}+\tau \sqrt{2 n} \sigma)$.

It is in fact better to use $w_{1}[n](n \bar{x}+\tau \sqrt{n} \sigma) \approx 2 w_{1}[4 n](4 n \bar{x}+2 \tau \sqrt{n} \sigma)$ which does not introduce new linking points and does not increase the complexity of the next computations. Starting from $n=31, w_{1}[32]$ may be approximated from $w_{1}[8]$ and so on until $w_{1}[124]$ from $w_{1}[31]$, which allows the computation of $\sum_{j=8}^{31} \rho^{4 j-1} c[4 j]$. Thereafter, a convolution with $\sum_{j=1}^{3} \rho^{k} c[k]$ allows the computation of the other terms (whose indices are not $4 n$ ).

\section{Conclusion}

In this paper, computing methods have been designed in order to evaluate response time of packet switching networks. Efficient algorithms have been proposed to study M/GI/1 queues. They use formal and thereafter numerical techniques. They decrease approximation errors for the set of values which are not small enough to use standard techniques and not large enough to use large deviation techniques. Examples were shown from M/D/1 queues, but the algorithms apply to the general case. A key point to conclude: the proposed method does not generate a Gibbs' phenomenon at discontinuity points (Fig. 2), unlike others algorithms based upon orthogonal functions, and the remaining error term is no longer an oscillating function.

\section{Acknowledgments}

Thanks to the anonymous referees for helpfull comments.

\section{References}

1. 1959. Gaver D. P. "Embedded Markov chain analysis of a waiting line process in continuous time", Annals Math. Stat. 30, 698-720

2. 1968. (Dubner-Abate algorithm) : Dubner H. and J. Abate. "Numerical inversion of Laplace transforms by relating them to finite Fourier-cosine transform", J. ACM 15(1):115-123.

3. 1971. (Salzer-Piessens algorithm) : Piessens, R. "Gaussian quadrature formulas for the numerical integration of Bromwich's integral and the inversion of the Laplace transform". J. Engineer. Math. 5(1):1-9.

4. 1970. (Gaver-Stehfest algorithm) : Stehfest H. "Algorithm 368 [D5] : Numerical inversion of Laplace transforms", Comm. ACM 13(1):47-49 and 13(10):624.

5. 1966. (Widder-Weeks algorithm) : Weeks W.T. "Numerical inversion of Laplace transforms using Laguerre functions", J. ACM. 13(3):419-426.

6. 1994. Cheng, A.H-D., P. Sidaurik and Y. Abousleiman. "Approximate inversion of the Laplace transform", The Mathematica Journal 4(2):76-82.

7. 1975. Kleinrock. "Queuing systems, Vol. 1: theory", Wiley

8. 1998. Douillet P.L. "A study of some queuing systems", Ph.D. Thesis, University of Paris, 253 p. Available at : www-inf. int-evry.fr/- douillet/thesis.

In 8], more than 200 references, are given concerning inverse Laplace Transform, queuing systems and formal calculus. 